\title{
MAMMALIAN MODELS FOR THE STUDY OF VOCAL LEARNING: A NEW PARADIGM IN BATS
}

\author{
ELLA Z. LATTENKAMP ${ }^{1,2}$, SONJA C. VERNES ${ }^{1, *, \dagger}$, and LUTZ WIEGREBE ${ }^{2, \dagger}$ \\ *Corresponding author: sonja.vernes@mpi.nl \\ ${ }^{1}$ Neurogenetics of Vocal Communication, Max Planck Institute for Psycholinguistics, \\ Nijmegen, The Netherlands \\ ${ }^{2}$ AG Wiegrebe, Dept. Biology II, Ludwig-Maximilians University, Munich, Germany \\ ${ }^{\dagger}$ Authors contributed equally
}

\section{Animal models for the study of human speech}

The complexity of human spoken language in structure and expression is unmatched in the animal kingdom. However, several animal species possess the capacity for vocal production learning (in the following simply referred to as 'vocal learning'), which is a requirement for the acquisition and production of human speech. Vocal learning is defined as the ability to imitate new sounds or modify existing signals from a preexisting repertoire according to auditory input (Boughman \& Moss, 2003; Janik \& Slater, 1997). Even though on the surface this may seem simple enough, this is a complex trait that requires the functional interplay of precise auditory perception, neuronal integration of the signal (including template memorization and matching), and accurate neuronal motor control of the vocal production systems (Fitch, 2000). In order to gain an indepth understanding of the evolution and the biological basis of human spoken language, in vivo experiments are an essential prerequisite. As these are challenging in humans, animal models are of great importance for the experimental approach to answering questions about the origins of human language and speech acquisition.

\subsection{Vocal Learning in vertebrates}

Vocal learning as a behavioural trait can be found in different levels of expression within the animal kingdom and should be regarded as a continuum (Petkov \& 
Jarvis, 2012). Several vertebrate species have shown indications for vocal learning in the past. These include three groups of birds (songbirds, hummingbirds, and parrots) and several mammalian taxa (cetaceans, pinnipeds, elephants, and bats). In the past decades, songbirds, which occupy a position toward the upper end of the vocal learning continuum, have been studied extensively and this research has yielded invaluable insights in the physiological prerequisites, neurobiology, and genetics required for vocal learning (Jarvis et al., 2005; Pfenning et al., 2014; Roberts et al., 2017; Wheatcroft \& Qvarnström, 2015). While research on avian model organisms dominates the field, mammalian vocal learning by comparison has largely been neglected in the past.

\subsection{Bats as a mammalian model for Vocal Learning}

Comparing the available sample sizes, ease of study, and potential different mammalian vocal learners provide, bats present themselves as a useful candidate model system for the study of vocal learning. Bats are gregarious, highly vocal animals with a broad vocal repertoire. In the past, several bats species have shown indications of vocal learning (Knörnschild, 2014; Prat, Taub \& Yovel, 2015). However, controlled, repeatable experiments with isolated adult bats have thus far been lacking.

We have developed an automated setup and a conditioning training regime on social vocalisations of isolated bats, which allow for the spectro-temporal tracking of call parameters and their changes over time. This approach for the first time enables the detailed study of vocal parameters and behaviours on an individual level. We were able to demonstrate the volitional use of communication calls out of context and in the absence of any social feedback, hence, capturing usage learning in isolated bats. Furthermore, by changing the reward schedule, we induced temporal and spectral adjustment of call parameters, showing vocal plasticity in bat social calls. These studies of individual bats will provide the basis for the in-depth study of vocal development, and vocal learning in a mammalian model species. Repeatable experiments on isolated individuals will further allow the investigation of common morphological structures among vocal learners, shared auditory processing pathways, and neuronal and gene networks underlying the mammalian vocal learning capacity.

Our experimental approach demonstrates the value of bats as a mammalian model system for vocal learning and will take us a step forward in understanding the evolution of human speech and language acquisition. 


\section{Acknowledgements}

This work was funded by a Human Frontiers Science Program (HFSP) Research grant (RGP0058/2016) awarded to L.W. and S.C.V. and a Max Planck Research Group Grant awarded to S.C.V.

\section{References}

Boughman, J.W. \& Moss, C.F. (2003). Social sounds: vocal learning and development of mammal and bird calls. In: A. M. Simmons, R. R. Fay, and A. N. Popper (Eds), Acoustic Communication - Springer Handbook of Auditory Research (pp. 138-224). Springer-Verlag: New York, Inc.

Fitch, W.T. (2000). The evolution of speech: a comparative review. Trends in Cognitive Sciences, 4, 258-267.

Janik, V.M. and Slater, P.J.B. (1997). Vocal learning in mammals. Advances in the Study of Behaviour, 26, 59-99.

Jarvis, E.D.; Güntürkün, O.; Bruce, L.; Csillag, A.; Karten, H.; Kuenzel, W.; Medina, L.; Paxinos, G.; Perkel, D.J.; Shimizu, T.; Striedter, G.; Wild, J.M.; Ball, G.F.; Dugas-Ford, J.; Durand, S.E.; Hough, G.E.; Husband, S.; Kubikova, L.; Lee, D.W.; Mello, C. V.; Powers, A.; Siang, C.; Smulders, T. V.; Wada, K.; White, S.A.; Yamamoto, K.; Yu, J.; Reiner, A. and Butler, A.B. (2005). Avian brains and a new understanding of vertebrate brain evolution. Nature Reviews Neuroscience, 6, 151-159.

Knörnschild, M. (2014). Vocal production learning in bats. Current Opinion in Neurobiology, 28, 80-85.

Petkov, C.I. and Jarvis, E.D. (2012). Birds, primates, and spoken language origins: behavioral phenotypes and neurobiological substrates. Frontiers in Evolutionary Neuroscience, 4, 1-24.

Pfenning, A.R.; Hara, E.; Whitney, O.; Rivas, M. V; Wang, R.; Roulhac, P.L.; Howard, J.T.; Wirthlin, M.; Lovell, P. V; Ganapathy, G.; Mouncastle, J.; Moseley, M.A.; Thompson, J.W.; Soderblom, E.J.; Iriki, A.; Kato, M.; Thomas, M.; Gilbert, P.; Zhang, G.; Bakken, T.; Bongaarts, A.; Bernard, A.; Lein, E.; Mello, C. V; Hartemink, A.J. and Jarvis, E.D. (2014). Convergent transcriptional specializations in the brains of humans and song-learning birds. Science, 346, 1-13.

Prat, Y.; Taub, M. and Yovel, Y. (2015). Vocal learning in a social mammal: demonstrated by isolation and playback experiments in bats. Science Advances, 1, 1-5.

Roberts, T.F.; Hisey, E.; Tanaka, M.; Kearney, M.G.; Chattree, G.; Yang, C.F.; Shah, N.M. and Mooney, R. (2017). Identification of a motor-to-auditory pathway important for vocal learning. Nature Neuroscience, 20, 978-986.

Wheatcroft, D. and Qvarnström, A. (2015). A blueprint for vocal learning: auditory predispositions from brains to genomes. Biology letters, 11, 20150155 . 